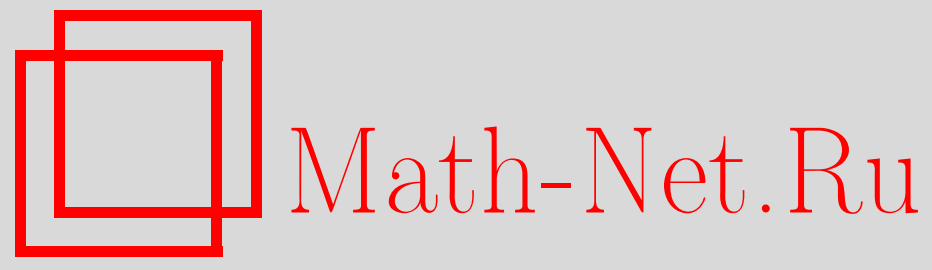

Я. А. Шахов, Многопрограммная стабилизация положений равновесия квазилинейных стационарных систем, Вестн. Сам. гос. техн. ун-та. Сер. Физ.-мат. науки, 2012, выпуск 1(), 46-51

DOI: https://doi.org/10.14498/vsgtu980

Использование Общероссийского математического портала Math-Net.Ru подразумевает, что вы прочитали и согласны с пользовательским соглашением

http://www.mathnet.ru/rus/agreement

Параметры загрузки:

IP: 54.80 .97 .219

26 апреля 2023 г., 12:41:39

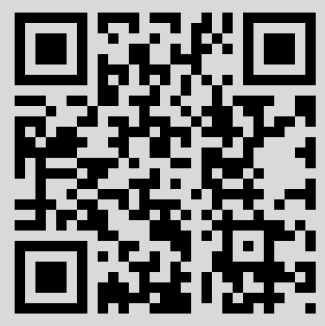


УДК 517.977.1, 519.71

\title{
МНОГОПРОГРАММНАЯ СТАБИЛИЗАЦИЯ ПОЛОЖЕНИЙ РАВНОВЕСИЯ КВАЗИЛИНЕЙНЫХ СТАЦИОНАРНЫХ СИСТЕМ
}

\author{
Я. А. Шахов \\ Санкт-Петербургский государственный университет, \\ 198504, Россия, Санкт-Петербург, Университетский просп., 35. \\ E-mail: yakov.shakhov@gmail.com
}

\begin{abstract}
Рассматривается задача многопрограммной стабилизащии положений равновесия квазилинейных стационарных систем. Положения равновесия являются важными (с точки зрения моделирования) режимами функиионирования любой динамической системы. Многопрограммное управление, реализующее данные режимь, строится в виде интерполячионного полинома Эрмита. Доказана теорема о достаточных условиях существования многопрограммного стабилизирующего управления, приведён иллюстративный пример.
\end{abstract}

Ключевые слова: квазилинейная система, стабилизация, многопрограммное управление, стачионарная система, положение равновесия.

Введение. Классическая задача стабилизации объекта на заданной траектории $[1,2]$ подразумевает конструирование некоторого стабилизирующего устройства, которое подавляет возникающие отклонения от программного движения объекта управления. В задаче многопрограммной стабилизации [3] предлагается строить управление, реализующее несколько программных режимов и обеспечивающее их асимптотическую устойчивость по Ляпунову $[4,5]$. В настоящей работе задача многопрограммной стабилизации формулируется для квазилинейной системы [4], в качестве программных режимов выступают естественные положения равновесия системы.

Постановка задачи. Рассмотрим квазилинейную стационарную систему [4] с управлением

$$
\dot{\mathbf{x}}=\mathbf{A x}+\mathbf{B u}+\mathbf{G}(\mathbf{x}, \mathbf{u})
$$

где $\mathbf{x}=\left(x_{1}, x_{2}, \ldots, x_{n}\right)^{\top}-n$-мерный вектор фазового состояния, $\mathbf{u}=\left(u_{1}, u_{2}\right.$, $\left.\ldots, u_{r}\right)^{\top}-r$-мерный вектор управлений; $\mathbf{A}=\left\{a_{i j}\right\}, \mathbf{B}=\left\{b_{i k}\right\}-$ постоянные матрицы, $i=1,2, \ldots, n, j=1,2, \ldots, n, k=1,2, \ldots, r ; \mathbf{G}(\mathbf{x}, \mathbf{u})$ - вещественная, непрерывно дифференцируемая по компонентам $\mathbf{x}$ и $\mathbf{u}$ вектор-функция, для которой справедливо

$$
\frac{\|\mathbf{G}(\mathbf{x}, \mathbf{u})\|}{\|\mathbf{x}\|} \rightrightarrows 0 \text { при }\|\mathbf{x}\| \rightarrow 0 .
$$

Пусть на систему (1) не оказывается никакого программного управляющего воздействия $\left(\mathbf{u}=\mathbf{u}_{p}(t)=\mathbf{0}\right)$.

ОПРЕДЕЛЕНИЕ 1 [5]. Точка $\mathbf{x}_{0}$ называется положением равновесия, или точкой покоя системъ (1), если есть частное решение системы $\mathbf{x}\left(t, \mathbf{x}_{0}\right)=\mathbf{x}_{0}$ при $t \in(-\infty,+\infty)$.

Яков Александрович Шахов, аспирант, каф. моделирования экономических систем. 
Найдём для (1) положения равновесия. Для этого решим нелинейную систему уравнений

$$
\mathbf{A x}+\mathbf{G}(\mathbf{x}, 0)=\mathbf{0}
$$

относительно $\mathbf{x}$. Будем считать, что таких точек $\mathbf{x}_{j}$ конечное число $N<+\infty$, то есть $j=1,2, \ldots, N$.

ЗАДАЧА (МНОГОПРОГРАММНАЯ СТАБИЛИЗАЦИЯ ПОЛОЖЕНИЙ РАВНОВЕСИЯ КВАЗИЛИНЕЙНЫХ СИСТЕМ). Для исходной системы (1) требуется построитъ многопрограммное управление $\mathbf{u}=\mathbf{u}(\mathbf{x})$ [3], которое обеспечивает асимптотическую устойчивость по Ляпунову положений равновесия $\mathbf{x}_{j}, j=1,2, \ldots, N$, системы.

ЗАмечАниЕ. В общем случае для системы (1) положения равновесия могут обеспечиваться ненулевым управляющим воздействием $\mathbf{u}_{p} \neq \mathbf{0}$. В этом случае совокупность положений равновесия исходной системы рассматривается как некоторое семейство программных режимов.

Решение задачи. Следуя результатам работы [6], будем строить решение поставленной задачи в виде интерполяционного полинома Әрмита следующего вида:

$$
\mathbf{u}(\mathbf{x})=\sum_{j=1}^{N} \mathbf{C}_{j}\left(\mathbf{x}-\mathbf{x}_{j}\right) p_{j}(\mathbf{x}), \quad p_{j}(\mathbf{x})=\prod_{i=1, i \neq j}^{N} \frac{\left(\mathbf{x}-\mathbf{x}_{i}\right)^{2}}{\left(\mathbf{x}_{j}-\mathbf{x}_{i}\right)^{2}}
$$

Здесь узловыми точками являются положения равновесия системы (1), а её значением - нулевые программные режимы. Матрица коэффициентов усиления стабилизирующего управления $\mathbf{C}_{j}, j=1,2, \ldots, N$, для каждого положения равновесия в общем случае своя $\left(\mathbf{C}_{j} \neq \mathbf{C}_{i}\right.$ при $\left.j \neq i\right)$.

Замкнём систему (1) управлением (3) и для $s$-ного положения равновесия $\mathbf{x}_{s}$ построим систему в отклонениях. Для $\mathbf{y}_{s}(t)=\mathbf{x}(t)-\mathbf{x}_{s}$ получим

$$
\dot{\mathbf{y}}_{s}=\mathbf{A} \mathbf{y}_{s}+\mathbf{B} \sum_{j=1}^{N} \mathbf{C}_{j}\left(\mathbf{y}_{s}+\mathbf{x}_{s}-\mathbf{x}_{j}\right) p_{j}\left(\mathbf{y}_{s}+\mathbf{x}_{s}\right)+\widetilde{\mathbf{Q}}_{s}\left(\mathbf{y}_{s}\right),
$$

где $\widetilde{\mathbf{Q}}_{s}\left(\mathbf{y}_{s}\right)=\mathbf{G}\left(\mathbf{y}_{s}+\mathbf{x}_{s}, \mathbf{u}\left(\mathbf{y}_{s}+\mathbf{x}_{s}\right)\right)-\mathbf{G}\left(\mathbf{x}_{s}, \mathbf{0}\right)$.

В системе (4) выделим линейное приближение. Заметим, что в $\widetilde{\mathbf{Q}}_{s}\left(\mathbf{y}_{s}\right)$ могут появляться линейные члены по $\mathbf{y}_{s}$, т. е.

$$
\widetilde{\mathbf{Q}}_{s}\left(\mathbf{y}_{s}\right)=\widetilde{\mathbf{A}}_{s} \mathbf{y}_{s}+\mathbf{Q}_{s}\left(\mathbf{y}_{s}\right),
$$

где слагаемое $\mathbf{Q}_{s}\left(\mathbf{y}_{s}\right)$ нелинейно по $\mathbf{y}_{s}$.

Также учтём свойства функций $p_{j}\left(\mathbf{y}_{s}+\mathbf{x}_{s}\right), j=1,2, \ldots, N$, из представления (4).

1) Если $j \neq s$, то в составе произведения

$$
p_{j}\left(\mathbf{y}_{s}+\mathbf{x}_{s}\right)=\prod_{i=1, i \neq j}^{N} \frac{\left(\mathbf{y}_{s}+\mathbf{x}_{s}-\mathbf{x}_{i}\right)^{2}}{\left(\mathbf{x}_{j}-\mathbf{x}_{i}\right)^{2}}, \quad j=1,2, \ldots, N,
$$


при $i=s$ будет сомножитель

$$
\frac{\mathbf{y}_{s}^{2}}{\left(\mathbf{x}_{j}-\mathbf{x}_{s}\right)^{2}}
$$

Это означает, что все слагаемые суммы в правой части (4) при $j \neq s$ имеют порядок не меньше второго по компонентам вектора $\mathbf{y}_{s}$ и, следовательно, не входят в систему линейного приближения.

2) Если $j=s$, то

$$
p_{s}\left(\mathbf{y}_{s}+\mathbf{x}_{s}\right)=\prod_{i=1, i \neq s}^{N} \frac{\left(\mathbf{y}_{s}+\mathbf{x}_{s}-\mathbf{x}_{i}\right)^{2}}{\left(\mathbf{x}_{s}-\mathbf{x}_{i}\right)^{2}}
$$

Очевидно, что каждый сомножитель в (5) имеет вид

$$
\frac{\left(\mathbf{y}_{s}+\mathbf{x}_{s}-\mathbf{x}_{i}\right)^{2}}{\left(\mathbf{x}_{s}-\mathbf{x}_{i}\right)^{2}}=\frac{\mathbf{y}_{s}^{2}}{\left(\mathbf{x}_{s}-\mathbf{x}_{i}\right)^{2}}+2 \frac{\left(\mathbf{x}_{s}-\mathbf{x}_{i}\right) \mathbf{y}_{s}}{\left(\mathbf{x}_{s}-\mathbf{x}_{i}\right)^{2}}+1, \quad i=1,2, \ldots, N, i \neq s .
$$

В результате (5) можно представить в виде

$$
p_{s}\left(\mathbf{y}_{s}+\mathbf{x}_{s}\right)=1+2 \sum_{i=1, i \neq s}^{N} \frac{\left(\mathbf{x}_{s}-\mathbf{x}_{i}\right) \mathbf{y}_{s}}{\left(\mathbf{x}_{s}-\mathbf{x}_{i}\right)^{2}}+h_{s}\left(\mathbf{y}_{s}\right)
$$

где $h_{s}\left(\mathbf{y}_{s}\right)$ - скалярная функция, являющаяся суммой слагаемых, порядок которых по компонентам вектора $\mathbf{y}_{s}$ не меньше двух.

Рассмотрим правые части системы (4) с учётом свойств 1), 2) функций $p_{j}\left(\mathbf{y}_{s}+\mathbf{x}_{s}\right), j=1,2, \ldots, N$. Очевидно, что дополнительные линейные члены по $\mathbf{y}_{s}$ могут появиться только при $j=s$. Рассмотрим отдельно слагаемое, соответствующее $j=s$, и учтём (6):

$$
\mathbf{B C}_{s} \mathbf{y}_{s}\left(1+2 \sum_{i=1, i \neq s}^{N} \frac{\left(\mathbf{x}_{s}-\mathbf{x}_{i}\right) \mathbf{y}_{s}}{\left(\mathbf{x}_{s}-\mathbf{x}_{i}\right)^{2}}+h_{s}\left(\mathbf{y}_{s}\right)\right)=\mathbf{B C}_{s} \mathbf{y}_{s}+\widetilde{\mathbf{H}}_{s}\left(\mathbf{y}_{s}\right),
$$

где

$$
\widetilde{\mathbf{H}}_{s}\left(\mathbf{y}_{s}\right)=\mathbf{B C}_{s} \mathbf{y}_{s}\left(2 \sum_{i=1, i \neq s}^{N} \frac{\left(\mathbf{x}_{s}-\mathbf{x}_{i}\right) \mathbf{y}_{s}}{\left(\mathbf{x}_{s}-\mathbf{x}_{i}\right)^{2}}+h_{s}\left(\mathbf{y}_{s}\right)\right)
$$

С учётом данного представления система (4) примет вид

$$
\dot{\mathbf{y}}_{s}=\left(\mathbf{A}_{s}+\mathbf{B C}_{s}\right) \mathbf{y}_{s}+\mathbf{H}_{s}\left(\mathbf{y}_{s}\right)+\mathbf{Q}_{s}\left(\mathbf{y}_{s}\right) .
$$

Здесь

$$
\mathbf{A}_{s}=\mathbf{A}+\widetilde{\mathbf{A}}_{s}, \quad \mathbf{H}_{s}\left(\mathbf{y}_{s}\right)=\widetilde{\mathbf{H}}_{s}\left(\mathbf{y}_{s}\right)+\mathbf{B} \sum_{j=1, j \neq s}^{N} \mathbf{C}_{j}\left(\mathbf{y}_{s}+\mathbf{x}_{s}-\mathbf{x}_{j}\right) p_{j}\left(\mathbf{y}_{s}+\mathbf{x}_{s}\right)
$$


Функция $\mathbf{H}_{s}\left(\mathbf{y}_{s}\right)$ имеет полиномиальный вид. Её порядок по компонентам вектора $\mathbf{y}_{s}$ не меньше второго, а максимальная степень конечна и зависит только от параметра $N$. Следовательно, при достаточно малых по норме отклонений $\mathbf{y}_{s}$ справедлива оценка

$$
\left\|\mathbf{H}_{s}\left(\mathbf{y}_{s}\right)\right\| \leqslant a\left\|\mathbf{y}_{s}\right\|^{b}
$$

где $b \geqslant 2, a$ - положительная константа, зависящая от норм матриц $\mathbf{B}, \mathbf{C}_{j}$ и векторов $\mathbf{x}_{j}, j=1,2, \ldots, N$.

Теперь, после предварительных рассуждений, можно сформулировать следующее утверждение

Теорема. Пусть для системы (1) выполнены следующие условия:

1) заданные положения равновесия системы $\mathbf{x}_{1}, \mathbf{x}_{2}, \ldots, \mathbf{x}_{N}$ различны: $\mathbf{x}_{j} \neq$ $\neq \mathbf{x}_{i}$ npu $j \neq i$

2) однороднъе системь $\dot{\mathbf{y}}_{j}=\mathbf{A}_{j} \mathbf{y}_{j}+\mathbf{B} \mathbf{v}_{j}$ при $\mathbf{v}_{j}=\mathbf{C}_{j} \mathbf{y}_{j}$ могут иметь сколь угодно большой запас устойчивости, получающийся путем выбора постоянных матрич $\mathbf{C}_{j}, j=1,2, \ldots, N$;

3) $\frac{\left\|\mathbf{Q}_{s}\left(\mathbf{y}_{s}\right)\right\|}{\left\|\mathbf{y}_{s}\right\|} \rightrightarrows 0$ nри $\left\|\mathbf{y}_{s}\right\| \rightarrow 0$ равномерно по $t \geqslant 0$.

Тогда существует многопрограммное управление, обеспечивающее каждому положению равновесия из семейства $\mathbf{x}_{1}, \mathbf{x}_{2}, \ldots, \mathbf{x}_{N}$ асимптотическую устойчивость по Ляпунову.

Доказ ательств в. Выше построена система в отклонениях (4), проанализированы свойства функции $\mathbf{H}_{s}\left(\mathbf{y}_{s}\right)$. Осталось показать асимптотическую устойчивость нулевого решения этой системы.

Согласно второму условию теоремы, за счёт стабилизирующего управления $\mathbf{v}_{s}=\mathbf{C}_{s} \mathbf{y}$ собственные числа матрицы $\mathbf{A}_{s}+\mathbf{B} \mathbf{C}_{s}$ можно сделать любыми наперед заданными, в том числе с отрицательными вещественными частями [1]. В этом случае нулевое решение каждой системы (4) при $s=1,2, \ldots, N$ асимптотически устойчиво по линейному приближению [4]. Действительно, с одной стороны, $\mathbf{H}_{s}\left(\mathbf{y}_{s}\right)$ неявно зависит от времени через $\mathbf{v}_{s}(t), \mathbf{y}_{s}(t), s=$ $=1,2, \ldots, N$, с другой стороны, в силу ограниченности этих функций и первого условия теоремы имеет место следующее свойство:

$$
\lim _{\left\|\mathbf{y}_{s}\right\| \rightarrow 0} \frac{\left\|\mathbf{H}_{s}\left(\mathbf{y}_{s}\right)\right\|}{\left\|\mathbf{y}_{s}\right\|}=0
$$

равномерно по $t \geqslant 0$. Следовательно, с учётом третьего условия теоремы, все положения равновесия системы (1) при управлении (3) будут асимптотически устойчивы по Ляпунову.

ПримеР. Решим задачу многопрограммной стабилизации положений равновесия для следующей квазилинейной системы

$$
\left\{\begin{array}{l}
\dot{x}_{1}=x_{2}+x_{3}+u, \\
\dot{x}_{2}=-x_{1}+x_{3}+\left(x_{1}^{2}-x_{2}^{2}\right), \\
\dot{x}_{3}=u+x_{1}^{2}
\end{array}\right.
$$

Представляя систему (7) в векторно-матричном виде (1), получим

$$
\mathbf{A}=\left(\begin{array}{ccc}
0 & 1 & 1 \\
-1 & 0 & 1 \\
0 & 0 & 0
\end{array}\right), \quad \mathbf{B}=(1,0,1)^{\top}, \quad \mathbf{G}(\mathbf{x}, \mathbf{u})=\left(0, x_{1}^{2}-x_{2}^{2}, x_{1}^{2}\right)^{\top}
$$


Решив нелинейную систему уравнений (2), найдём положения равновесия

$$
\mathbf{x}_{1}=(0,0,0)^{\top}, \quad \mathbf{x}_{2}=(0,-1,1)^{\top} .
$$

Многопрограммное стабилизирующее управление для системы (7) будем строить в виде

$$
\mathbf{u}(\mathbf{x})=\mathbf{C}_{1}\left(\mathbf{x}-\mathbf{x}_{1}\right) \frac{\left(\mathbf{x}-\mathbf{x}_{2}\right)^{2}}{\left(\mathbf{x}_{1}-\mathbf{x}_{2}\right)^{2}}+\mathbf{C}_{2}\left(\mathbf{x}-\mathbf{x}_{2}\right) \frac{\left(\mathbf{x}-\mathbf{x}_{1}\right)^{2}}{\left(\mathbf{x}_{2}-\mathbf{x}_{1}\right)^{2}}
$$

Пусть $\mathbf{y}_{s}=\left(y_{s 1}, y_{s 2}, y_{s 3}\right)^{\top}=\mathbf{x}-\mathbf{x}_{s}, s=1,2$. Для системы (7), замкнутой многопрограммным управлением (8), построим системы в отклонениях:

$$
\dot{\mathbf{y}}_{s}=\left(\mathbf{A}_{s}+\mathbf{B C}_{s}\right) \mathbf{y}_{s}+\mathbf{H}_{s}\left(\mathbf{y}_{s}\right)+\mathbf{Q}_{s}\left(\mathbf{y}_{s}\right), \quad s=1,2,
$$

где для $s=1$

$$
\begin{gathered}
\mathbf{A}_{1}=\mathbf{A}, \quad \mathbf{Q}_{1}\left(\mathbf{y}_{1}\right)=\left(0, y_{11}^{2}-y_{12}^{2}, y_{11}^{2}\right)^{\top}, \\
\mathbf{H}_{1}\left(\mathbf{y}_{1}\right)=\mathbf{B}\left\{\mathbf{C}_{1} \mathbf{y}_{1}\left(\mathbf{y}_{1} \mathbf{x}_{2}+\frac{\mathbf{y}_{1}^{2}}{2}\right)+\mathbf{C}_{2}\left(\mathbf{y}_{2}-\mathbf{x}_{2}\right) \frac{\mathbf{y}_{1}^{2}}{2}\right\} ;
\end{gathered}
$$

для $s=2$

$$
\begin{gathered}
\mathbf{A}_{2}=\left(\begin{array}{rrr}
0 & 1 & 1 \\
-1 & 2 & 1 \\
0 & 0 & 0
\end{array}\right), \quad \mathbf{Q}_{2}\left(\mathbf{y}_{2}\right)=\left(0, y_{21}^{2}-y_{22}^{2}-1, y_{21}^{2}\right)^{\top}, \\
\left.\mathbf{H}_{2}\left(\mathbf{y}_{2}\right)=\mathbf{B}\left\{\mathbf{C}_{1} \mathbf{y}_{2}+\mathbf{x}_{2}\right) \frac{\mathbf{y}_{2}^{2}}{2}+\mathbf{C}_{2} \mathbf{y}_{2}\left(\mathbf{y}_{2} \mathbf{x}_{2}+\frac{\mathbf{y}_{2}^{2}}{2}\right)\right\}
\end{gathered}
$$

Исследуем систему линейного приближения для (7) на полную управляемость. Для этого построим матрицы Калмана [5]:

$$
\mathbf{S}_{1}=\left\{\mathbf{B}, \mathbf{A}_{1} \mathbf{B}, \mathbf{A}_{1}^{2} \mathbf{B}\right\}=\left(\begin{array}{rrr}
1 & 1 & 0 \\
0 & 0 & -1 \\
1 & 0 & 0
\end{array}\right), \mathbf{S}_{2}=\left\{\mathbf{B}, \mathbf{A}_{2} \mathbf{B}, \mathbf{A}_{2}^{2} \mathbf{B}\right\}=\left(\begin{array}{rrr}
1 & 1 & 0 \\
0 & 0 & -1 \\
1 & 0 & 0
\end{array}\right) .
$$

Они имеют полный ранг $\left(\operatorname{rank} S_{1}=3, \operatorname{rank} S_{2}=3\right)$, следовательно, системы линейного приближения для (7) полностью управляемы, а значит, и стабилизируемы [5].

Управление (8), согласно доказанной выше теореме, является многопрограммным стабилизирующим для исходной системы (7) и обеспечивает асимптотическую устойчивость положений равновесия $\mathbf{x}_{1}, \mathbf{x}_{2}$. Матрицы коэффициентов усиления стабилизирующего управления $\mathbf{C}_{1}, \mathbf{C}_{2}$ могут быть найдены по известным алгоритмам [2] как стабилизирующие матрицы соответствующих систем линейного приближения.

Заключение. Таким образом, изучен вопрос построения многопрограммного стабилизирующего управления для квазилинейной стационарной системы, обеспечивающего асимптотическую устойчивость ее положений равновесия. Результаты продемонстрированы на численном примере. 


\section{БИБЛИОГРАФИЧЕСКИЙ СПИСОК}

1. Зубов В. И. Лекции по теории управления. М.: Наука, 1975. 495 с. [Zubov V. I. Lectures in control theory. Moscow: Nauka, 1975. 495 pp.]

2. Смирнов Н.В., Смирнова Т.Е., Тамасян Г.Ш. Стабилизация программных движений в пространстве состояний. СПб.: Соло, 2010. 97 с. [Smirnov N. V, Smirnova T.E., Tamasyan G. Sh. The stabilization of program motions in the state space. St. Petersburg: Solo, 2010. 97 pp.]

3. Зубов В.И. Синтез многопрограммных устойчивых управлений// Докл. АН СССР, 1991. Т. 318, № 2. С. 274-277; англ. пер.: Zubov V. I. Synthesis of multiprogram stable controls // Soviet Math. Dokl., 1991. Vol.43, no. 3. Pp. 689-692.

4. Демидович Б. П. Лекции по математической теории устойчивости. М.: Наука, 1967. 472 c. [Demidovich B. P. Lectures on the mathematical theory of stability. Moscow: Nauka, 1967. 472 pp.]

5. Александров А.Ю., Александрова В.М., Екимов А.В., Смирнов Н.В. Сборник задач и упражнений по теории устойчивости. Иркутск: Иркут. гос. ун-т, 2008. 162 с. [Aleksandrov A. Yu., Aleksandrova V.M., Ekimov A.V., Smirnov N.V. Collection of problems and exercises on the stability theory. Irkutsk: Irkut. Gos. Un-t, 2008. 162 pp.]

6. Смирнов Н. В., Шахов Я. А. Многопрограммная стабилизация квазилинейных систем // Вестн. С.-Петерб. ун-та. Сер. 10. Прикладная математика, информатика, прочессы управления, 2010. №4. С. 128-138. [Smirnov N. V., Shakhov Ya.A. Multiprogram stabilization of quasi-linear systems // Vestn. St. Petersburg Univ., Ser. 10. Applied Mathematics. Computer Science. Control Processes, 2010. no. 4. Pp. 128-138].

Поступила в редакцию 16/VII/2011;

в окончательном варианте - 13/IX/2011.

\section{MSC: $34 \mathrm{H} 15$}

\section{MULTIPROGRAMMED STABILIZATION OF THE EQUILIBRIUM POSITIONS OF THE QUASI-LINEAR TIME-INVARIANT SYSTEMS}

\section{Y. A. Shakhov}

St. Petersburg State University,

35, Universitetskiy prosp., St. Petersburg, 198504, Russia.

E-mail: yakov.shakhov@gmail.com

In present work, the problem of multiprogrammed stabilization of the equilibrium positions for a quasi-linear system is considered. The equilibrium positions are very important (from the viewpoint of dynamic object simulation) functioning regimes of any dynamic system. The multiprogrammed controls which realized these regimes are constrained as the Hermit's interpolating polynomials. In the paper, the theorem on suffcient conditions of the multiprogrammed stabilized control existence is proved and the illustrative example is given.

Key words: quasi-linear system, stabilization, multiprogrammed control, time-invariant dynamic system, equilibrium position. 